\title{
Modification of the Lagrange Interpolating Polynomial Scheme for Using with the Finite Difference Method
}

\author{
Uthai Prasopchingchana ${ }^{1, a}$ \\ ${ }^{1}$ Department of Mechanical Engineering, Burapha University, Chonburi 20131, Thailand
}

\begin{abstract}
Modification of the Lagrange interpolating polynomial (LIP) scheme for using with the finite difference method is proposed. Merits of the modified LIP scheme used with the finite difference method for problem solving are facile to discretize equations, and fast to obtain solutions. Verification of the modified LIP scheme was performed by comparison of the solutions computed from the modified LIP scheme with the analytical solutions of a heat conduction problem. The verification gives credence to the modified LIP scheme for correctness of the solutions. In addition, the comparison results of solution accuracy and computational time of problem solving between using of the finite difference method with the modified LIP scheme and the finite volume method with the LIP scheme are exhibited.
\end{abstract}

\section{Introduction}

Most scientific and engineering problems are defined by partial difference equations. There are many numerical methods for solution determination of the problems such as the finite difference method (FDM), the finite volume method (FVM), the finite element method, the boundary element method and the lattice Boltzmann method etc. The FDM is not complicated, easy to understand and develop codes, and has low cost in memory and computational time. Problem solving by using high-order schemes is a means which high accurate solutions are desired. But, using the FDM with existing highorder schemes and non-uniform grids is quite burdensome.

In the last decade, there were many authors devising new high-order schemes not difficult to solve problems by using with the FDM and non-uniform grids. Filbet and Prouveur [1] developed a temporal high-order scheme for backward semi-Lagrangian methods and applied to transport equations for plasma physics problems. In addition, Chen et al. [2], Zhao and Deng [3], Wang et al. [4], Baeza et al. [5], and Jha and Kumar [6] constructed spatial high-order schemes for using with the FDM and non-uniform grids, and performed verification of their schemes with different problems.

Recently, the Lagrange interpolating polynomial (LIP) scheme for using with the FVM was proposed by Prasopchingchana and Manewattana [7]. The LIP scheme is easily modified for using with the FDM, called the modified LIP scheme.

An aim of this article is to propose a modified LIP scheme for using with the FDM. The modified LIP scheme is a high-order scheme used for discretization of the first and second derivatives of variables with respect to both space and time. Using the modified LIP scheme with the FDM to solve problems simulated by using non-uniform grids is facile, and solution convergence of the using is fast.

${ }^{\text {a }}$ Corresponding author : uthai@buu.ac.th 


\section{Modification of the LIP scheme for using with the finite difference method}

From Figure 1., the first derivative values of variables with respect to time can be approximated by using the modified LIP scheme and can be expressed as

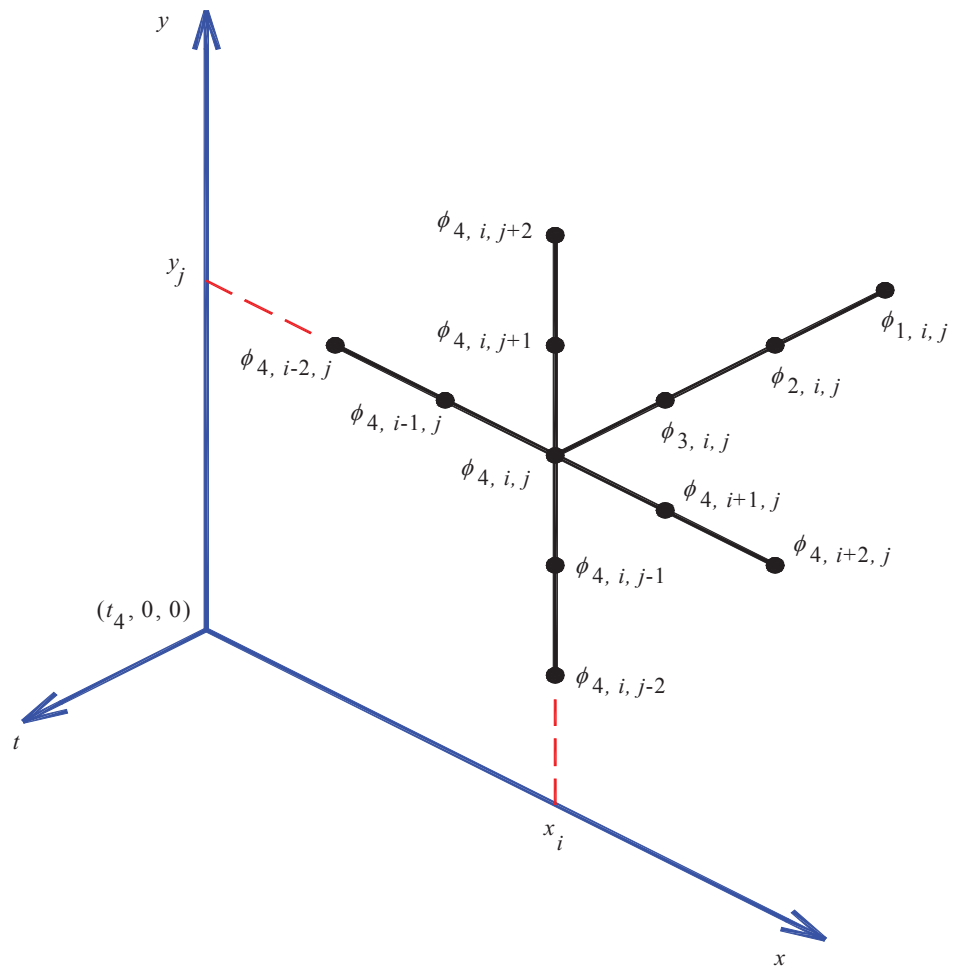

Figure 1. Schematic details of the modified LIP scheme for using with FDM.

$$
\frac{d \phi_{4, i, j}}{d t}=\sum_{n t=1}^{4}\left(D L T_{n t} \phi_{n t, i, j}\right)
$$

where $\phi, t$ and $D L T$ are a variable, time and the first temporal derivative coefficients of the modified LIP scheme. The first temporal derivative coefficients in Equ. (1) can be computed from

$$
D L T_{n t}=\frac{d}{d T T}\left(\prod_{\substack{m=1 \\ m \neq n t}}^{4} \frac{\left(T T-T T_{m}\right)}{\left(T T_{n t}-T T_{m}\right)}\right)
$$

where $T T_{n t}=t_{n t}-t_{4}, T T_{m}=t_{m}-t_{4}$ and $T T=0$

The first and second derivative values of variables with respect to space in the horizontal direction can be approximated by using the modified LIP scheme and can be written as

$$
\begin{aligned}
& \frac{d \phi_{4, i, j}}{d x}=\sum_{n x=1}^{5}\left(D L X_{n x, i} \phi_{4, i-n x h+n x, j}\right) \\
& \frac{d^{2} \phi_{4, i, j}}{d x^{2}}=\sum_{n x=1}^{5}\left(D D L X_{n x, i} \phi_{4, i-n x h+n x, j}\right)
\end{aligned}
$$


where $x, D L X, D D L X$ and $n x h$ are space in the horizontal direction, the first and second spatial derivative coefficients of the modified LIP scheme and a node constant value in the horizontal direction, respectively. The first and second spatial derivative coefficients in Equ. (3a) and (3b) can be calculated from

$$
\begin{aligned}
& D L X_{n x, i}=\frac{d}{d X}\left(\prod_{\substack{i i=1 \\
i i \neq n x}}^{5} \frac{\left(X-X_{i i}\right)}{\left(X_{n x}-X_{i i}\right)}\right) \\
& D D L X_{n x, i}=\frac{d^{2}}{d X^{2}}\left(\prod_{\substack{i i=1 \\
i i \neq n x}}^{5} \frac{\left(X-X_{i i}\right)}{\left(X_{n x}-X_{i i}\right)}\right)
\end{aligned}
$$

where $X_{n x}=x_{i-n x h+n x}-x_{i}, X_{i i}=x_{i-n x h+i i}-x_{i}$ and $X=0$. And the node constant value in the horizontal direction is $n x h=1$ for $i=$ imin , $n x h=2$ for $i=i m i n+1$, $n x h=3$ for $i m i n+2 \leq i \leq \operatorname{imax}-2, n x h=4$ for $i=i \max -1$ and $n x h=5$ for $i=i m a x$ where imin and imax are the minimum and maximum numbers of nodes in the horizontal direction.

Equ. (2), (4a) and (4b) can be simplified by changing to be algebraic forms. The algebraic forms of the first and second, temporal and spatial derivative coefficients of the modified LIP scheme are shown in Table 1.

\begin{tabular}{|c|c|c|}
\hline First derivative coefficient & Second derivative coefficient & Remark \\
\hline$D L T_{n t}=\frac{\sum_{\substack{m=1 \\
m \neq n t}}^{4} \prod_{\substack{m m=1 \\
m m \neq m \\
m m \neq n t}}^{4}\left(-T T_{m m}\right)}{\prod_{\substack{m=1 \\
m \neq n t}}^{4}\left(T T_{n t}-T T_{m}\right)}$ & - & $\begin{aligned} T T_{n t} & =t_{n t}-t_{4} \\
T T_{m} & =t_{m}-t_{4} \\
T T_{m m} & =t_{m m}-t_{4}\end{aligned}$ \\
\hline$D L X_{n x, i}=\frac{\sum_{\substack{i=1 \\
i=1 \\
i \neq n x}}^{5} \prod_{\substack{i i i=1 \\
i i i \neq i i \\
i i i \neq n x}}^{5}\left(-X_{i i i}\right)}{\prod_{\substack{i i=1 \\
i i \neq n x}}^{5}\left(X_{n x}-X_{i i}\right)}$ & $D D L X_{n x, i}=\frac{2 \sum_{\substack{i i=1 \\
i i \neq n x}}^{5}\left(X_{i i}\left(\sum_{\substack{i i i=1 \\
i i+i i i \neq n x}}^{5-i i}\left(X_{i i+i i i}\right)\right.\right.}{\prod_{\substack{i i=1 \\
i i \neq n x}}^{5}\left(X_{n x}-X_{i i}\right)}$ & $\begin{aligned} X_{n x} & =x_{i-n x h+n x}-x_{i} \\
X_{i i} & =x_{i-n x h+i i}-x_{i} \\
X_{i i i} & =x_{i-n x h+i i i}-x_{i}\end{aligned}$ \\
\hline$D L Y_{n y, j}=\frac{\sum_{\substack{j j=1 \\
j j \neq n y}}^{5} \prod_{\substack{j i j=1 \\
j j j \neq j j \\
j j j \neq n y}}^{5}\left(-Y_{j j j}\right)}{\prod_{\substack{j j=1 \\
j j \neq n y}}^{5}\left(Y_{n y}-Y_{j j}\right)}$ & $D D L Y_{n y, j}=\frac{2 \sum_{\substack{j j=1 \\
j j \neq n y}}^{5}\left(Y_{j j}\left(\sum_{\substack{j j j=1 \\
j j+j j j \neq n y}}^{5-j j}\left(Y_{j j+i j j}\right)\right.\right.}{\prod_{\substack{j j=1 \\
j j \neq n y}}^{5}\left(Y_{n y}-Y_{j j}\right)}$ & $\begin{aligned} Y_{n y} & =y_{j-n y h+n y}-y_{j} \\
Y_{j j} & =y_{j-n y h+j j}-y_{j} \\
Y_{j j j} & =y_{j-n y h+j j j}-y_{j}\end{aligned}$ \\
\hline
\end{tabular}

Table 1. The first and second, temporal and spatial derivative coefficients of the modified LIP scheme. 


\section{Verification of the modified LIP scheme}

Verification of the modified LIP scheme for using with the FDM must be performed for confidence that the modified LIP scheme works correctly. Comparison of the solutions computed from the modified LIP scheme with the analytical solutions of a "heat conduction in rectangular plates with boundary temperatures specified" problem was adopted for the verification. The analytical solutions reported by Beck et al. [8] are the well-known solutions of the problem. An in-house code was developed based on how to solve the problem by using the modified LIP scheme with the FDM.

For the problem, interior temperatures in the rectangular plates with the specified boundaries as shown in Figure 2. can be calculated from

$$
\rho c \frac{\partial T}{\partial t}-k\left(\frac{\partial^{2} T}{\partial x^{2}}+\frac{\partial^{2} T}{\partial y^{2}}\right)=0
$$

where $\rho, c, k$ and $T$ are density, specific heat, thermal conductivity, and temperatures, respectively.

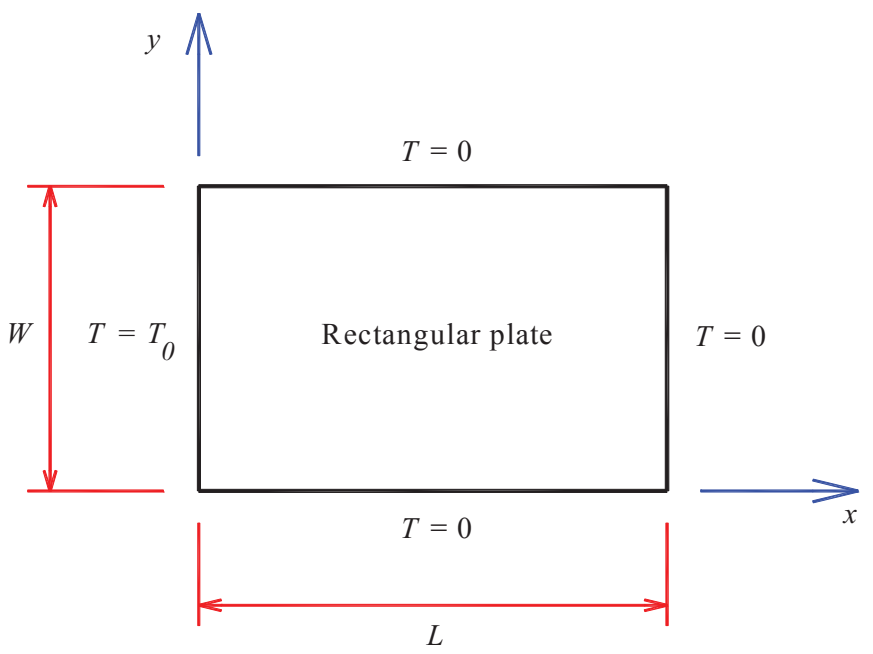

Figure 2. Schematic details of a "heat conduction in rectangular plates with boundary temperatures specified" problem for verification.

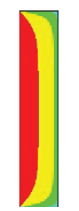

(a)

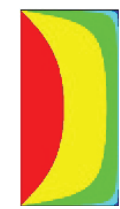

(b)

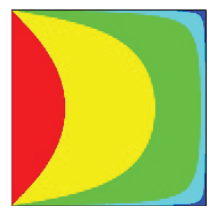

(c)

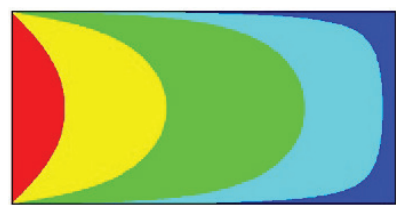

(d)

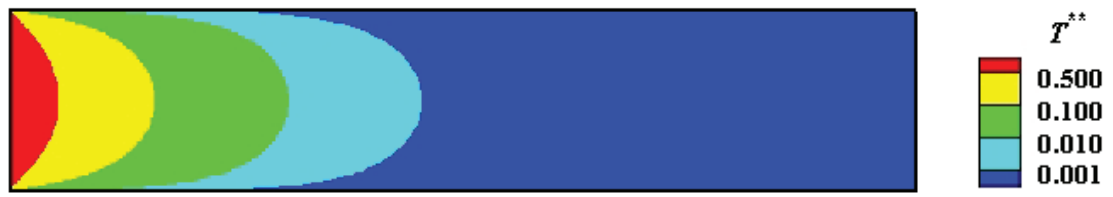

(e)

Figure 3. Dimensionless temperature contours of the solutions of a "heat conduction in rectangular plates with boundary temperatures specified" problem which are computed from the modified LIP scheme, (a) case 1, (b) case 2 , (c) case 3 , (d) case 4 and (e) case 5. 
Table 2. Comparison of the solutions computed from the modified LIP scheme with the analytical solutions of a "heat conduction in rectangular plates with boundary temperatures specified" problem.

\begin{tabular}{|c|c|c|c|c|c|c|c|}
\hline Case & Author & $x^{* *}$ & $y^{* *}$ & $L^{* *}$ & $\begin{array}{l}T\left(x^{* *}, y^{* *}\right) / T_{0} \\
\text { (Accuracy, \%) }\end{array}$ & $\begin{array}{c}q_{x}^{\prime \prime}\left(x^{* *}, y^{* *}\right) L / k T_{0} \\
(\text { Accuracy, \%) }\end{array}$ & $\begin{array}{c}q_{y}^{\prime \prime}\left(x^{* *}, y^{* *}\right) L / k T_{0} \\
\text { (Accuracy, \%) }\end{array}$ \\
\hline \multirow[b]{2}{*}{1} & [8] & 0.10 & 0.25 & 0.20 & 0.4874535168 & 0.9992238948 & -0.0393751511 \\
\hline & Present work & 0.10 & 0.25 & 0.20 & $\begin{array}{c}0.4875203277 \\
(99.9862938931)\end{array}$ & $\begin{array}{c}0.9992157526 \\
(99.9991851476)\end{array}$ & $\begin{array}{c}-0.0391183171 \\
(99.3477256777)\end{array}$ \\
\hline \multirow[b]{2}{*}{2} & [8] & 0.25 & 0.25 & 0.50 & 0.3640566638 & 0.9169912516 & -0.3798302130 \\
\hline & Present work & 0.25 & 0.25 & 0.50 & $\begin{array}{c}0.3641939947 \\
(99.9622776030)\end{array}$ & $\begin{array}{c}0.9166666901 \\
(99.9646058237)\end{array}$ & $\begin{array}{c}-0.3790895581 \\
(99.8050036899)\end{array}$ \\
\hline \multirow[b]{2}{*}{3} & {$[8]$} & 0.50 & 0.25 & 1.00 & 0.1820283319 & 0.6387957290 & -0.5371610386 \\
\hline & Present work & 0.50 & 0.25 & 1.00 & $\begin{array}{c}0.1822103823 \\
(99.8999878766)\end{array}$ & $\begin{array}{c}0.6387135936 \\
(99.9871421495)\end{array}$ & $\begin{array}{c}-0.5374608753 \\
(99.9441812271)\end{array}$ \\
\hline \multirow[b]{2}{*}{4} & [8] & 1.00 & 0.25 & 2.00 & 0.0388578672 & 0.2453678480 & -0.2435418264 \\
\hline & Present work & 1.00 & 0.25 & 2.00 & $\begin{array}{c}0.0389736704 \\
(99.7019826142)\end{array}$ & $\begin{array}{c}0.2453441945 \\
(99.9903599839)\end{array}$ & $\begin{array}{c}-0.2442754813 \\
(99.6987560983)\end{array}$ \\
\hline \multirow{2}{*}{5} & [8] & 2.50 & 0.25 & 5.00 & 0.0003495056 & 0.0054900240 & -0.0054900207 \\
\hline & Present work & 2.50 & 0.25 & 5.00 & $\begin{array}{c}0.0003561433 \\
(98.1008315747)\end{array}$ & $\begin{array}{c}0.0054902856 \\
(99.9952349935)\end{array}$ & $\begin{array}{c}-0.0055946527 \\
(98.0941419766)\end{array}$ \\
\hline
\end{tabular}

$\underline{\underline{\text { Note }}}$

Accuracy $=\left(1-\left|\frac{[8]-\text { Present work }}{[8]}\right|\right) \times 100$

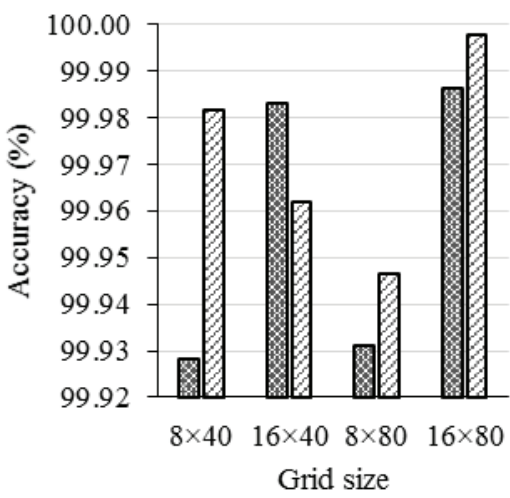

(a)

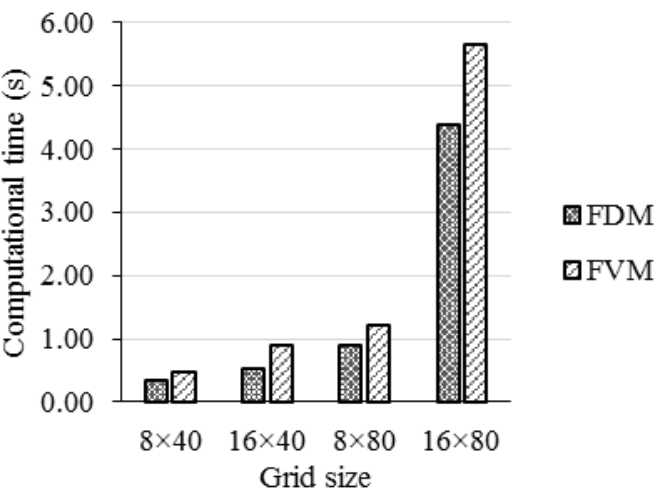

(b)

Figure 4. Comparison of solution accuracy and computational time of problem solving between using of the FDM with the modified LIP scheme and the FVM with the LIP scheme at the different grid sizes for case 1, (a) solution accuracy and (b) computational time. 


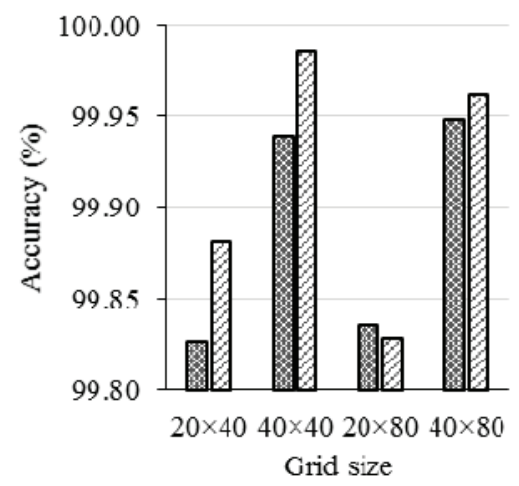

(a)

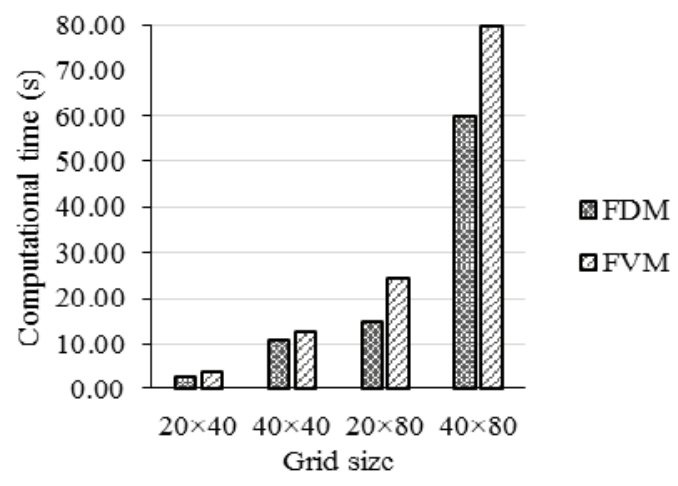

(b)

Figure 5. Comparison of solution accuracy and computational time of problem solving between using of the FDM with the modified LIP scheme and the FVM with the LIP scheme at the different grid sizes for case 2, (a) solution accuracy and (b) computational time.

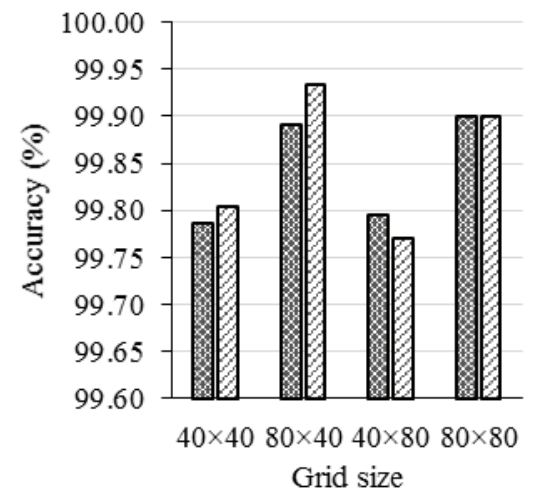

(a)

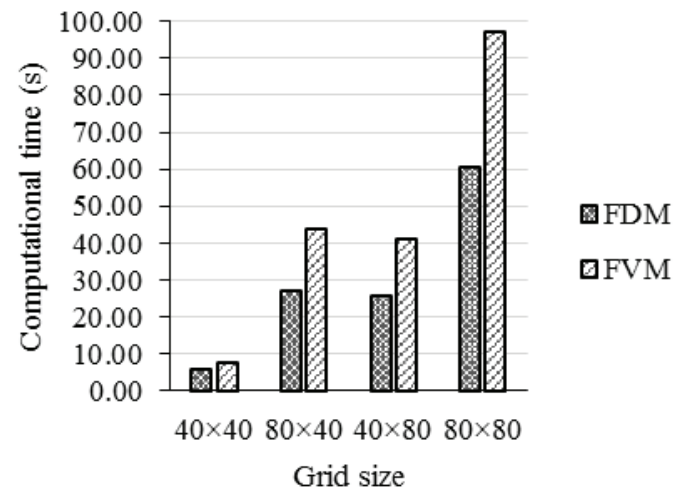

(b)

Figure 6. Comparison of solution accuracy and computational time of problem solving between using of the FDM with the modified LIP scheme and the FVM with the LIP scheme at the different grid sizes for case 3, (a) solution accuracy and (b) computational time.

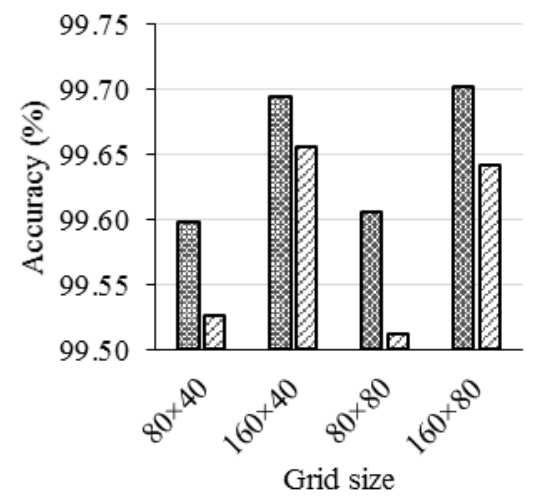

(a)

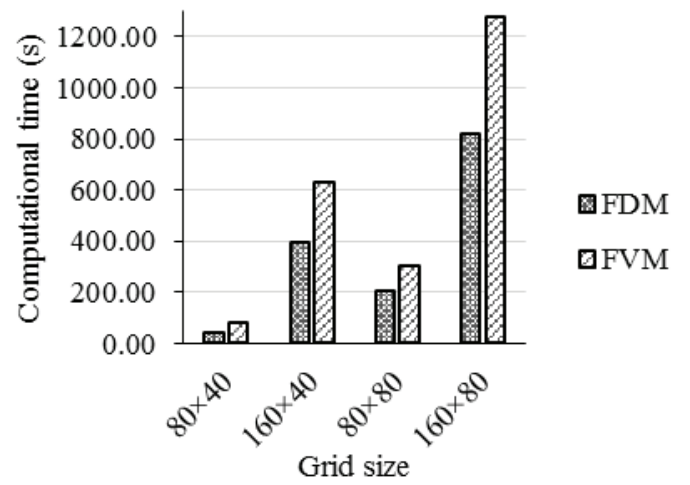

(b)

Figure 7. Comparison of solution accuracy and computational time of problem solving between using of the FDM with the modified LIP scheme and the FVM with the LIP scheme at the different grid sizes for case 4, (a) solution accuracy and (b) computational time. 


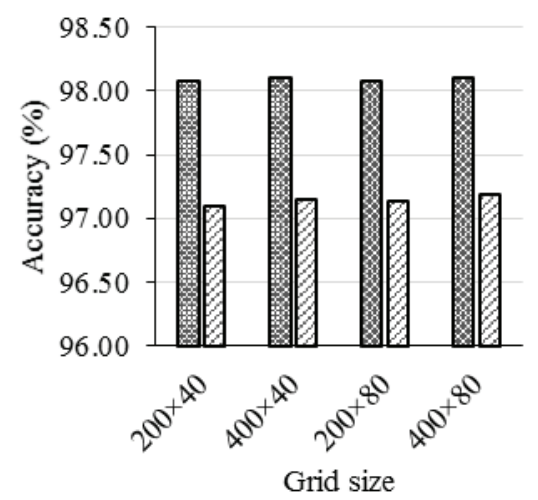

(a)

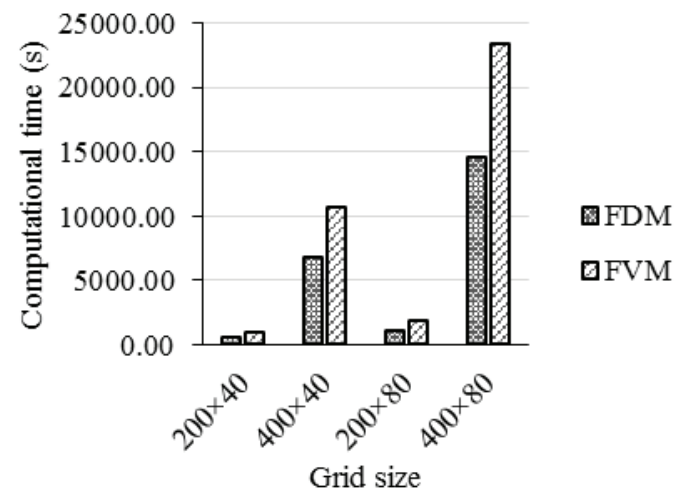

(b)

Figure 8. Comparison of solution accuracy and computational time of problem solving between using of the FDM with the modified LIP scheme and the FVM with the LIP scheme at the different grid sizes for case 5, (a) solution accuracy and (b) computational time.

The first temporal and second spatial derivative values of the temperatures in Equ. (5) can be discretized by using the modified LIP scheme.

$$
\rho c\left(\sum_{n t=1}^{4}\left(D L T_{n t} T_{n t, i, j}\right)\right)-k\left(\left(\sum_{n x=1}^{5}\left(D D L X_{n x, i} T_{4, i-n x h+n x, j}\right)\right)+\left(\sum_{n y=1}^{5}\left(D D L Y_{n y, j} T_{4, i, j-n y h+n y}\right)\right)\right)=0
$$

Equ. (6) can be rearranged to be

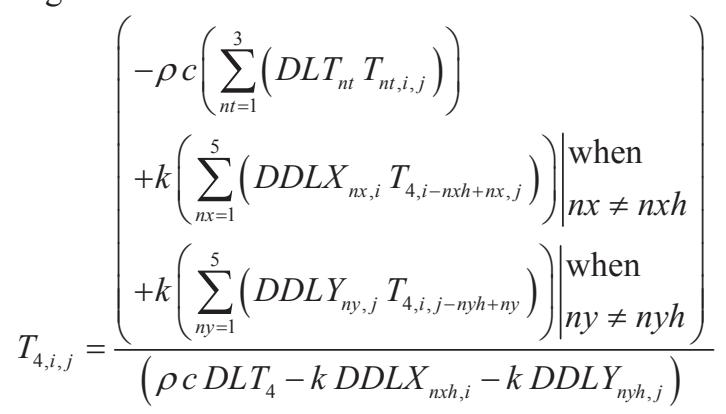

The Gauss-Seidel iterative method with the non-uniform grids were used to solve the equation system of Equ. (7). Fine grids were drawn near the boundaries of the rectangular plates, due to the steep temperature gradients. And coarse grids were applied in an interior area of the rectangular plates. A spatial ratio of the coarse grids to the fine grids was 1.05. A relative error of the code was less than or equal to $10^{-10}$, and a time step value of the code was $0.01 \mathrm{~s}$.

Table 2. shows comparison of the solutions computed from the modified LIP scheme with the analytical solutions of the "heat conduction in rectangular plates with boundary temperatures specified" problem. There are 5 cases of the difference of the dimensionless parameters for the comparison. For the present work, the modified LIP scheme which is the spatial $4^{\text {th }}$ order (a 9 nodes, $2 \mathrm{D}$ stencil) and the 4 time steps is adopted for discretization. The dimensionless parameters displayed in Table 2. can be calculated from $x^{* *}=x / W, y^{* *}=y / W$ and $L^{* *}=L / W$. The accuracies of the solutions computed from the modified LIP scheme can add credence to the modified LIP scheme.

Figure 3. illustrates dimensionless temperature contours of the solutions of the "heat conduction in rectangular plates with boundary temperatures specified" problem which are computed from the modified LIP scheme for 5 cases. The contour values in Figure 3. are specified according to the contour values shown in [8], and the contour pattern displayed in Figure 3. is similar to the contour pattern exhibited in [8]. 
Figure 4.-8. display comparison of the solution accuracies and computational time of the problem solving between using the FDM with the modified LIP scheme and using the FVM with the LIP scheme at different grid sizes for 5 cases. CPU time of the computer is adopted to be the computational time of the code. Specification of the computer used for code computation is "Intel ${ }^{\circledR}$ Core TM i5-4460 CPU @ 3.20 GHz 3.20 GHz". For the comparison of the solution accuracy, it can not specify which of the FDM or the FVM can give more accurate solutions. But, for the comparison of the computational time, the computational time of the problem solved by using the FDM with the modified LIP scheme is less than the computational time of the problem solved by using the FVM with the LIP scheme in all of the grid sizes and cases.

\section{Conclusion}

Modification of the LIP scheme for using with the FDM is presented. And, verification of the modified LIP scheme was performed. Advantages of the modified LIP scheme for using with the FDM are that it is a high-order scheme, easy to understand and develop codes, and spends less computational time for problem solving than by using the FVM with the LIP scheme.

\section{Acknowledgment}

The author wishes to acknowledge the Faculty of Engineering, Burapha University for the financial support to attend D2ME 2017.

\section{References}

1. F. Filbet, C. Prouveur, J. Comput. Appl. Math., 303, 171 (2016)

2. W. Chen, J. C. Chen, E. Y. Lo, Comput. Fluids, 101, 273 (2014)

3. L. Zhao, W. Deng, Adv. Comput. Math., 42, 425 (2016)

4. S. Wang, K. Virta, G. Kreiss J. Sci. Comput., 68, 1002 (2016)

5. A. Baeza, P. Mulet, D. Zorío, J. Sci. Comput., 69, 170 (2016)

6. N. Jha, N. Kumar, Adv. Differ. Equ. 64, 1 (2017)

7. U. Prasopchingchana, T. Manewattana, Eng. J., 19, 133 (2015)

8. J. V. Beck, N. T. Wright, A. Haji-Sheikh, K. D. Cole, D. E. Amos, Int. J. Heat Mass Transf., 51, $4676(2008)$ 